\title{
AERODYNAMIC STUDY OF MOTORCYCLE RACING WHEELS: A PERFORMANCE EVALUATION BASED ON NUMERICAL CFD SIMULATIONS
}

\author{
F. $\mathrm{CONCLI}^{1}, \mathrm{M} . \mathrm{GOBBI}^{2} \&$ C. GORLA ${ }^{2}$ \\ ${ }^{1}$ Free University of Bolzano/Bozen, Faculty of Science and Technology, Bolzano, Italy. \\ ${ }^{2}$ Politecnico di Milano, Department of Mechanical Engineering, Milano, Italy.
}

\begin{abstract}
In any racing competitions, the aerodynamic performances of the equipment are determinant. This is true, for example, for cars, where the geometry of the bodywork and of the wings can ensure a lower $\mathrm{Cx}$ coefficient and/or a higher down-force and a higher handling. In other competitions, like rowing, the aerodynamics of the hull can reduce the effort done by the athletes.

In the cycle and motorcycle racing competitions, other aspects related to aerodynamics become important, such as the manoeuvrability and stability.

In the present research, a numerical approach was used in order to compare different front-wheel geometries (of a racing motor-bike) in terms of drag, lift and axial forces. Three different wheel designs have been compared. The first one consists in a traditional seven spokes aluminium design, the second wheel is a 6 spokes magnesium solution and the third a solid-disk wheel.

Steady state as well as transient simulations was performed with OpenFOAM ${ }^{\circledR}$, a free open-source software. This was selected because it allows a higher flexibility with respect to any close-source commercial software. The possibility to customize the solver as well as the boundary conditions allows the analysis of the physical problem of interest. The free license allows a high parallelization of the computations.
\end{abstract}

The steady-state simulations were performed by freezing the wheel position and introducing a rotating reference frame. In this way, the computational time was significantly reduced.

For the transient simulations, the computational domain was split into two subdomains. The internal one is cylindrical and contains the wheel. The rotational velocity of the wheel was imposed by applying a rigid rotation to the mesh of the internal subdomain. Mesh interfaces ensures the continuity of the solution across the domains.

Keywords: CFD, Motorcycle, MRF, performance, OpenFOAM ${ }^{\circledR}$, wheel.

\section{INTRODUCTION}

The aerodynamic of most of the sport equipment is fundamental for the victory; racing teams are aware of this and put a lot of effort in the shape optimization. Small time differences on each lap, in fact, can affect significantly the race results.

Experimental tests have to be performed in wind-tunnels. Their costs can act as a deterrent to an experimental-driven aerodynamic design of vehicles. However, in the recent years, the development in computer science and the availability of more and more accurate numerical approaches are helping in overcoming this problem.

In the specific sector of motorbike racing, the main contributions to aerodynamic forces are given by the pilot, the motorcycle fairing and the wheels. Burke [1] and Lukes et al. [2] investigate the relative weight of the different parts of the motorbike to the total aerodynamic resistance. Greenwell et al. [3] showed that the wheels contribute to the total drag approximately by $10-15 \%$. An optimization seems to be possible. Zdravkovich [4] explained how, with proper wings, a drag reduction up to $5 \%$ could be easily obtained. Tew and Sayers [5] analysed the performance of traditional and optimized designs. A decrease of the drag up to $50 \%$ seems to be possible. Kyle and Burke [6-8] reported a considerable difference in the 
drag between rotating and stationary wheels. Similar behaviour has been measured by Fackrell and Harvey [9-11] for car tyres.

Despite the recent development in computer science have opened huge opportunities in terms of numerical simulation and more and more sectors started to use numerical tools such CAD, only few examples of numerical studies of the flow patterns around wheels rotating on the ground are available in actual scientific literature. Numerical analysis was performed by Wray [12] to quantify the influence of the yaw angle on the aerodynamic forces (lift and drag). The data published by Fackrell and Harvey were used from McManus et al. [13] to validate the results of their CFD simulations. Godo et al. [14] has measured a transition from a down- to a lift-force for a yaw angle between 5 and 8 degrees. Godo $e t$ al. [15] published a complete analysis of the flows around bike wheels that includes the resistant forces.

The authors have already presented a preliminary study in this field [16].

In this paper, the authors performed a CFD study (with OpenFOAM@ [17]) of the aerodynamic forces on a motorbike wheel subjected to different operating conditions. Three different wheel geometries were studied with steady state and dynamic approaches.

The steady-state simulations take advantage of a multireference frame (MRF) [18] coupled with an incompressible flow solver. The transient simulations were performed thanks to a partitioning strategy coupled with arbitrary interfaces (AMI) [19]. In this way, it was possible to simulate the problem just by applying a rigid rotation to the grid avoiding the need of remeshing (time demanding).

\section{WHEELS CONFIGURATIONS}

Three front wheel designs were studied. A standard geometry (namely: 'REF7') with seven spokes was used as reference. Other two wheels ('MGD6') with six spokes and with a solid-disk design ('DSKW') were considered. The tyre was the same for each wheel (Fig. 1).

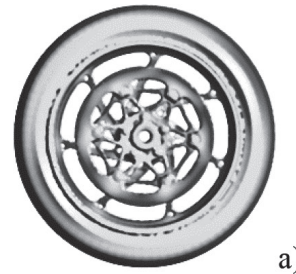

a)

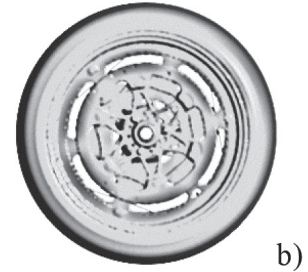

b)

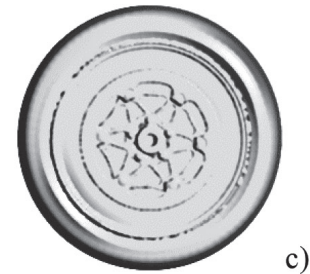

c)

Figure 1: Different configurations: (a) seven spokes REF7; (b) six spokes MGD6; and (c) solid disk design DSKW.

\section{SET-UP OF THE NUMERICAL ANALYSIS}

The wheels were modelled in contact with the road (ground).

The enclosing domain (a box) was selected after some preliminary test to ensure a good compromise between the computational effort (minimization of the surrounding volume) and the accuracy of the results (the domain have to be sufficiently large to including the fully developed aerodynamic wake).

Both for the steady state and for the transient simulations, the same geometrical model was used. The computational domain was subdivided creating a cylindrical partition that contains the wheel rim (not the tyre) as schematized in Fig. 2. 


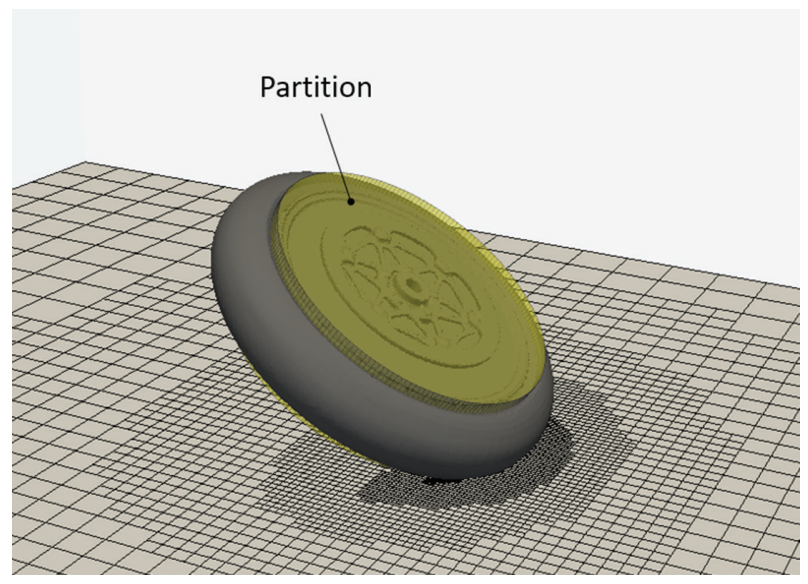

Figure 2: Internal partition.

A boundary layer was created on the 'Ground', the 'Wheel' and the 'Tyre' boundaries. The height of the 1st cell was chosen to have an $y^{+}$value between 50 and 500. The velocity profile on the boundaries was described with wall functions.

A velocity profile applied on the 'Inlet' and to the 'Ground' boundaries was applied and represents the speed of the motorbike. On the 'Outlet' boundary, the pressure was set equal to the ambient pressure. 'Tyre' and 'Wheel' boundaries were set as walls. Their velocities are referred to the rotating reference frame (MRF or rotating mesh in transient simulations). The value 0 reported in Table 1 refers to the relative reference frame. The reference speed of the bike was $60 \mathrm{~m} / \mathrm{s}$ that, for a tyre radius of $0.299 \mathrm{~m}$, corresponding to a rotational velocity of $0.05 \mathrm{rad} / \mathrm{s}$. However, simulations were performed in the whole range $30-120 \mathrm{~m} / \mathrm{s}$.

\subsection{Multi reference frame-steady-state approach}

In the steady approximation, individual cell zones ( 2 sub-domains) have different rotational velocities [20]. Equations in each cell zone are solved in the rotating reference frame.

Table 1: BC.

\begin{tabular}{|c|c|c|c|c|c|c|}
\hline & Ground & $\begin{array}{l}\text { Sides/ } \\
\text { Top }\end{array}$ & Inlet & Outlet & $\begin{array}{l}\text { Wheel } \\
\text { MRF/AMI }\end{array}$ & $\begin{array}{l}\text { Tyre } \\
\text { MRF/AMI }\end{array}$ \\
\hline $\mathrm{U}$ & $\begin{array}{l}\text { fixedValue } \\
\text { unif. 30-120 } \\
\mathrm{m} / \mathrm{s}\end{array}$ & symmetry & $\begin{array}{l}\text { fixedValue } \\
\text { unif. 30-120 } \\
\mathrm{m} / \mathrm{s}\end{array}$ & $\begin{array}{l}\text { zeroGradi- } \\
\text { ent }\end{array}$ & $\begin{array}{l}\text { moving- } \\
\text { WallVelocity } \\
\text { unif. } 0 \mathrm{~m} / \mathrm{s}\end{array}$ & $\begin{array}{l}\text { movingWall- } \\
\text { Velocity } \\
\text { unif. } 0 \mathrm{~m} / \mathrm{s}\end{array}$ \\
\hline $\mathrm{p}$ & zeroGradient & symmetry & zeroGradient & $\begin{array}{l}\text { fixedValue } \\
\text { unif.1E5Pa }\end{array}$ & $\begin{array}{l}\text { zeroGradi- } \\
\text { ent }\end{array}$ & zeroGradient \\
\hline eps & $\begin{array}{l}\text { epsilonWall- } \\
\text { Function }\end{array}$ & symmetry & fixedValue & inletOutlet & $\begin{array}{l}\text { epsilonWall- } \\
\text { Function }\end{array}$ & $\begin{array}{l}\text { epsilonWall- } \\
\text { Function }\end{array}$ \\
\hline $\mathrm{k}$ & $\begin{array}{l}\text { kqRWall- } \\
\text { Function }\end{array}$ & symmetry & fixedValue & inletOutlet & $\begin{array}{l}\text { kqRWall- } \\
\text { Function }\end{array}$ & $\begin{array}{l}\text { kqRWall- } \\
\text { Function }\end{array}$ \\
\hline
\end{tabular}


Therefore, the conservation equation includes the Coriolis $\left(2 \omega \times v_{r}\right)$ and the centripetal $(\omega \times \omega \times \omega)$ accelerations resulting in:

$$
\frac{\partial}{\partial t}\left(\rho v_{r}\right)+\nabla \cdot\left(\rho v_{r} v_{r}\right)+\rho\left(2 \omega \times v_{r}+\omega \times \omega \times r\right)=-\nabla_{p}+\nabla \cdot\left[\mu\left(\nabla v_{r}+\nabla v_{r}^{T}\right)\right]+\rho g+F
$$

$p$ represents the pressure, $\rho$ represents the density, $\mu$ represents the viscosity, $g$ represents the gravitational force per unit volume, $F$ represents the resultant of the external body forces per unit volume, $\omega$ represents the angular velocity referred to a stationary (inertial) reference frame and $v_{r}=\boldsymbol{v}-\omega \times \boldsymbol{r}$, where $r$ is the position vector from the origin of the rotating frame.

The continuity equation written in terms of relative velocities is given as

$$
\frac{\partial \rho}{\partial t}+\nabla \cdot\left(\rho v_{r}\right)=0
$$

At the common surfaces between the two cell zones (interfaces), a local reference frame transformation is performed in order to calculate fluxes across the surfaces. The Multi Reference Frame approach does not account for any relative motion of a moving zone with respect to its adjacent zones: the grid that remains does not move during computations. It is like freezing the motion of the body in a specific position and observing the instantaneous flowfield from a rotating reference system. Although the MRF approach is an approximation, it ensures quite accurate results for many configurations, ensuring at the same time, a considerably reduced computational effort with respect to any other FV approach. However, the MRF approach is not able to simulate the physical start-up giving just the regime solution.

\subsection{RMM and AMI—transient approach}

For the transient simulations, the same mesh was used but a different approach was applied. The portion of the grid that belongs to the internal cylindrical partition (yellow cylinder in Fig. 2) is set into rotation (rigid mesh motion-RMM). In this way, it is possible to correctly describe the physical start-up having an effective motion of the boundaries belonging to the wheel rim. For the rotating tyre, the imposition of a tangential velocity is enough to reproduce the motion, thanks to its axial-symmetry. On the other hand, this approach is less computationally efficient with respect to the MRF one [20]. This is because the whole physical transitory is simulated and also because the mesh motion implies additional calculations.

The (two) partitions have a mesh interface called AMI. AMI is a technique that allows simulation across disconnected, but adjacent, mesh domains. AMI operates by projecting one of the patches geometry onto the other (Fig. 3). In the present study, the interface has a cylindrical shape. The cell zone containing the wheel boundaries slides with a rigid motion along the mesh interface in discrete steps. The arbitrary mesh interface (AMI) projects the patches of the boundary mesh of one partition onto the other ensuring continuity of the internal fields.

\subsection{Other approaches}

Another option is to have a unique partition. The boundaries corresponding to wheel and tyre should be moved. This necessarily implies a grid distortion. When the quality of the mesh results is no more acceptable, the mesh should be updated/substituted [21]. This approach is 

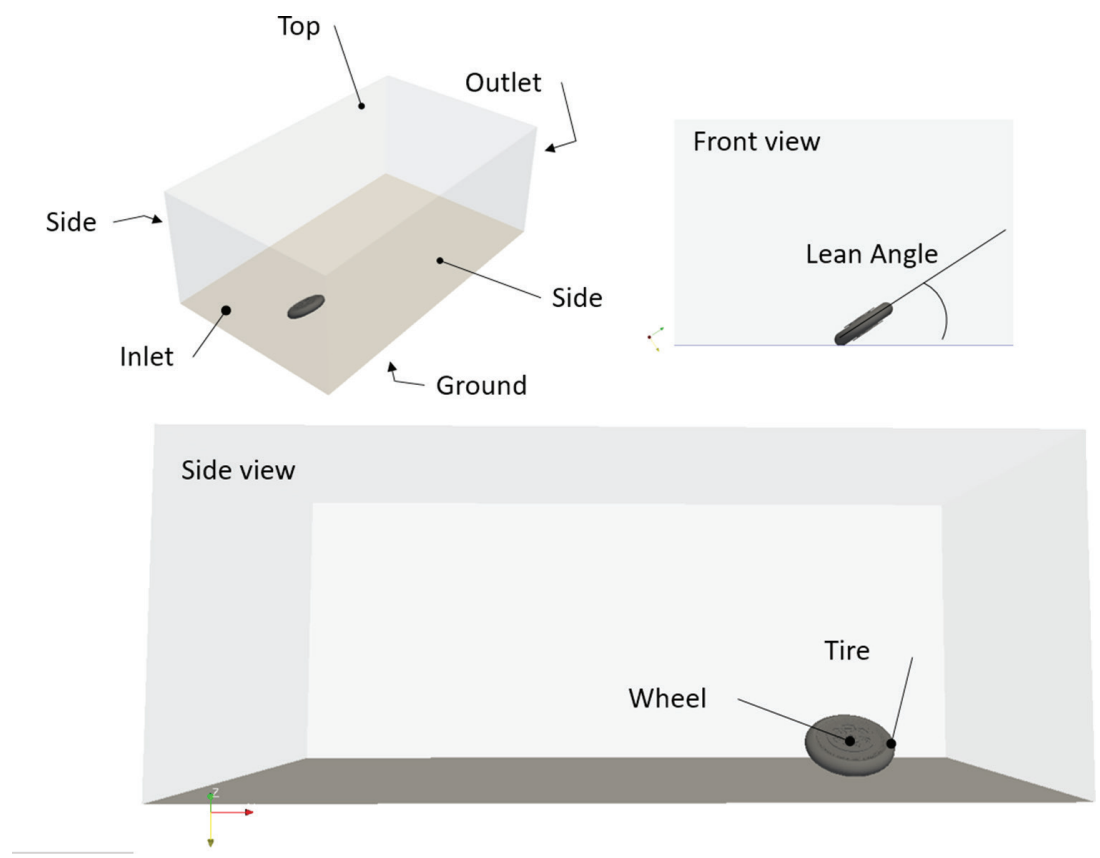

Figure 3: Patches.

very powerful and applicable to any geometry and motion, but also computationally demanding. An improvement in the simulation time can be achieved by updating only part of the grid (taking advantage of partitioning as in MRF and ADI) [22] or with the adoption of advanced mesh-handling algorithms [23-24]. In any case, this technique could never reach the computational efficiency of the RMM-AMI approach.

\section{NUMERICAL SETTINGS}

If the flows are compressible, the unknowns are the velocity vector and the density. The pressure is calculated using the constitutive equation. In the presence of incompressible flows, the pressure is not explicitly defined in any equation. If a pressure equation substitutes the continuity equation, after some mathematical manipulations, the pressure appears as unknown in the momentum equation and can be calculated.

The SIMPLE scheme was adopted to solve the pressure-velocity coupling in the steady calculations. For transient ones, a PIMPLE scheme was adopted. Further details about these schemes can be found in [25].

\section{RESULTS}

The aerodynamic forces acting on the different wheel geometries and velocities are summarized in Table 2 (steady-state calculations) in terms of drag forces (// to the travelling velocity), lift forces $(\perp$ to the axis of the wheel and to the travelling direction) and lateral forces (// to the wheel axis) [26]. The values between brackets represent the inertial and viscous contribution, respectively. Figure 8 reports the forces obtained from the transient analysis (performed only for the reference velocity of $60 \mathrm{~m} / \mathrm{s}$ ).

The velocity contour plot on the axial symmetry plane of the wheel and the vorticity are shown in Figs 4 and 5. Vorticity is defined as 
Table 2: Drag and lift—steady-state analysis.

\begin{tabular}{llll}
\hline & \multicolumn{3}{c}{ Total (inertial, viscous) forces 30 m/s } \\
& REF7 & MGD6 & DSKW \\
\hline Drag [N] (y) & $22.55(20.97,1.58)$ & $21.28(19.56,1.72)$ & $18.05(16.77,1.73)$ \\
Lift [N] (x) & $-7.01(-6.97,-0.03)$ & $-2.37(-2.47,0.10)$ & $-6.43(-6.41,-0.22)$ \\
Axial [N] (z) & $-4.22(-4.39,0.17)$ & $-12.37(-12.33,-0.04)$ & $-7.97(-8.18,0.21)$ \\
\hline \multicolumn{4}{c}{ Total (inertial, viscous) forces 60 m/s } \\
\hline & \multicolumn{4}{c}{ MGD6 } & DSKW \\
& REF7 & $93.32(87.57,5.75)$ & $72.15(66.185 .97)$ \\
\hline Drag [N] (y) & $90.14(84.68,5.46)$ & $-24.26(-24.63,0.47)$ & $-27.89(-28.590 .69)$ \\
Lift [N] (x) & $-32.08(-31.93,0.15)$ & $-73.64(-73.46,-0.18)$ & $-15.62(-15.52,-0.10)$ \\
\hline Axial [N] (z) & $-13.14(-13.73,-0.58)$ &
\end{tabular}

Total (inertial, viscous) forces $120 \mathrm{~m} / \mathrm{s}$

\begin{tabular}{lccc} 
& REF7 & MGD6 & DSKW \\
\hline Drag [N] (y) & $322.62(304.51,18.11)$ & $357.25(336.51$, & $273.39(250.40,22.99)$ \\
& & $20.74)$ \\
Lift [N] (x) & $-89.76(-91.03,1.27)$ & $-84.71(-86.76,2.05)$ & $-98.60(-101.56,2.96)$ \\
Axial [N] (z) & $-140.34(-139.67$, & $-296.87(-297.31$, & $-107.74(-107.39$, \\
& $-0.67)$ & $0.44)$ & $-0.35)$ \\
\hline
\end{tabular}
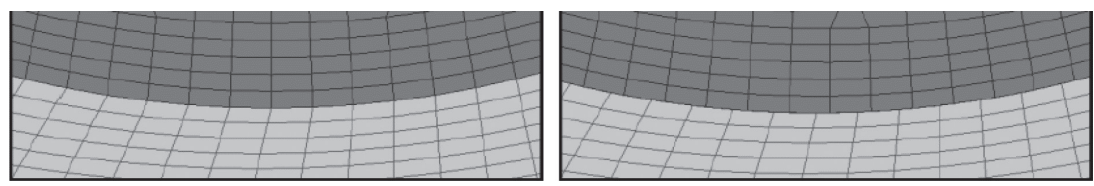

Figure 4: Sliding of the cells of the internal partition along the mesh interface [22].

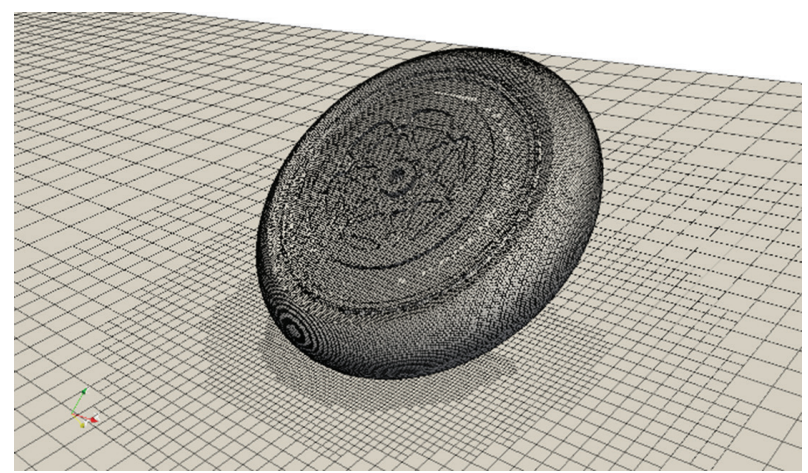

Figure 5: Mesh: wheel and ground. 


$$
\bar{\varpi}=\nabla \times u
$$

where $\mathrm{u}$ is the velocity vector. It describes the local spinning. An increase in the vorticity is related to a reduction of the drag forces. This is because a shifting downstream of the flow separation point corresponds to a higher vorticity [27].

At the reference speed of $60 \mathrm{~m} / \mathrm{s}$, a slight increase of the drag was observed for the MGD6 wheel. The DSKW configuration instead, is associated with a significant reduction of the drag force. In terms of lift, a reduction was observed from the seven spokes design (REF7) to the solid disk design (DSKW). The maximum gain was achieved with the MGD6 design (Figs 6 and 7)

Inertial effects are the main contribution to the drag, the lift and the axial forces. Viscous effects are comparable for the different geometries, but significantly lower with respect to the inertial contributions.

The frontal surface is equal to $0.09 \mathrm{~m}^{2}$ (for each design). The drag coefficients [28] result therefore 0.45 for the REF7 geometry, 0.47 for the MGD6 geometry and 0.36 for the DSKW one. The lift coefficients $0.16,0.12$ and 0.14 , respectively (Fig. 8).

At a higher speed $(120 \mathrm{~m} / \mathrm{s})$, the best performing solution in terms of drag results still the DSKW one (0.34), while the difference between the REF7 (0.40) and the MGD6 (0.45) becomes higher. The lift coefficients results in $0.11,0.10$ and 0.12 , respectively. Therefore, the benefits shown by the DSKW design at low speeds disappear at $120 \mathrm{~m} / \mathrm{s}$ and this solution becomes the less performing.

For the lowest speed $(30 \mathrm{~m} / \mathrm{s})$, the results show the same trends with drag coefficients equal to $0.45,0.43$ and 0.36 and lift ones equal to $0.14,0.05,0.13$. In these conditions, the lift of the MGD6 design is particularly low.

The effect of the rotational speed (travelling speed) on the forces is shown, for each geometry, in Fig. 9.

Figure 10 shows the influence of the geometries on the drag, lift and axial forces.
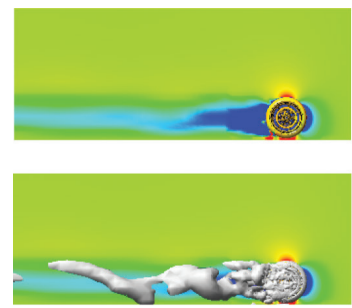

a)
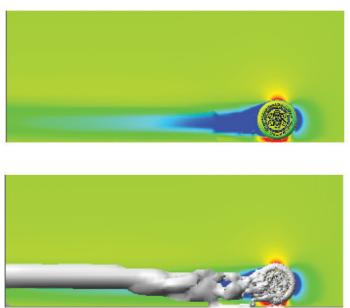

b)
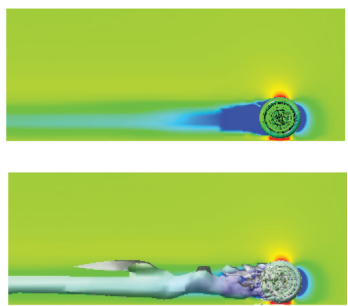

c)

Figure 6: velocity and vorticity plots—mid plane of the wheel: (a) REF7; (b) MGD6; (c) DSKW (60 m/s).

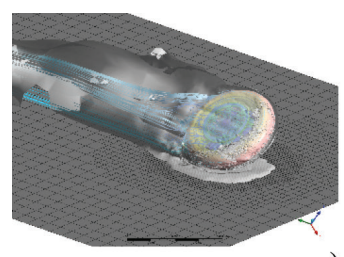

a)

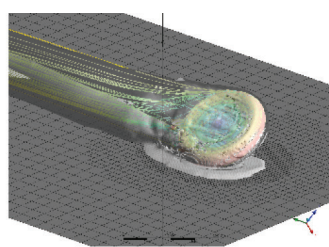

b)

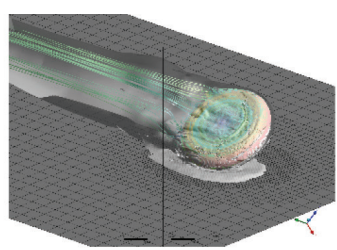

c)

Figure 7: Streamlines: (a) REF7; (b) MGD6; (c) DSKW (60 m/s). 

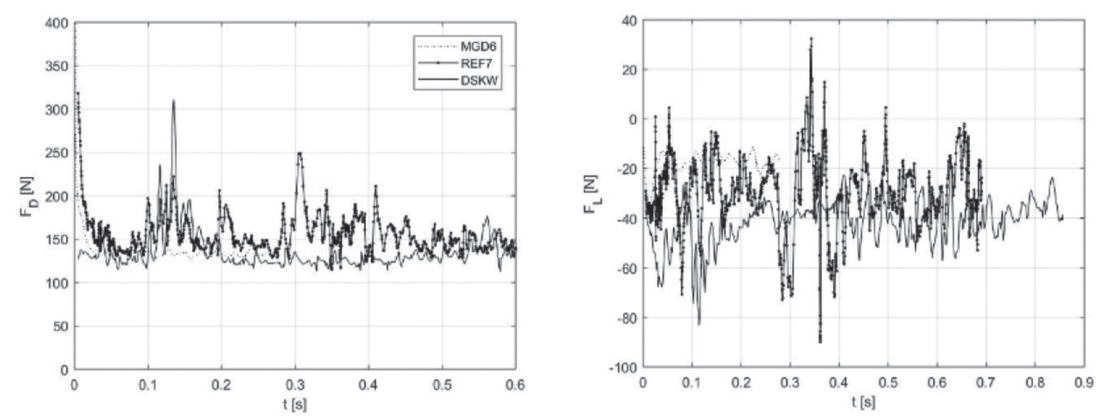

Figrue 8: Drag and lift—-transient calculations $(60 \mathrm{~m} / \mathrm{s})$.

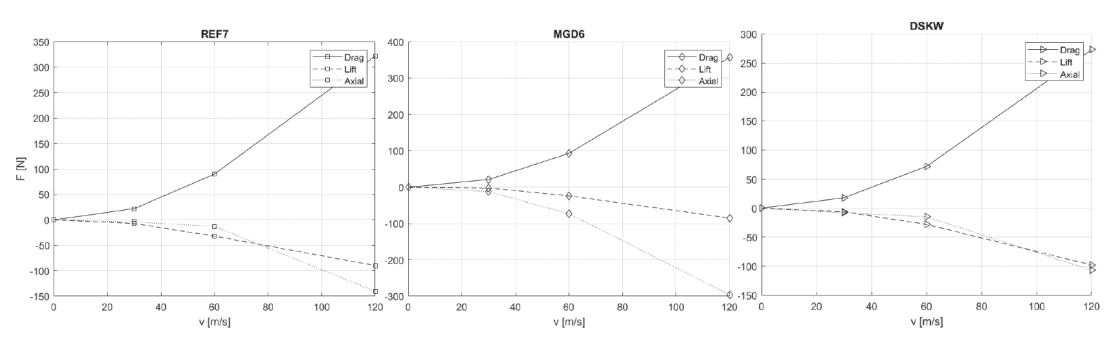

Figure 9: Influence of the travelling speed on the forces.
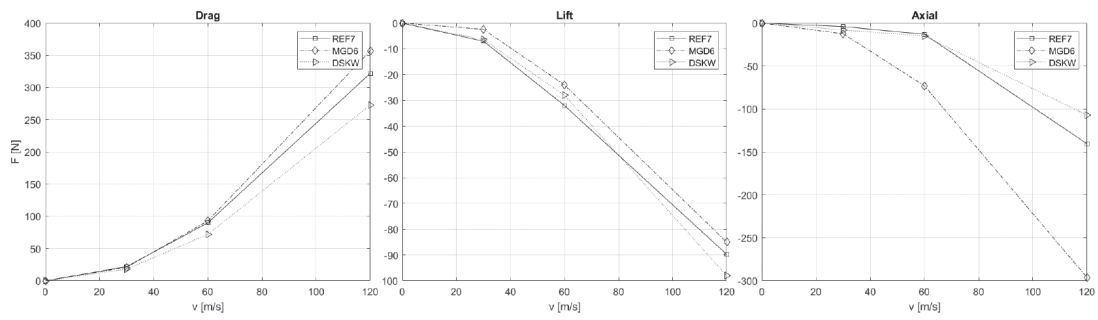

Figure 10: Drag, lift and axial forces on the different wheels as a function of the speed.

\section{CONCLUSIONS}

Three racing motorbike wheel have been analysed in terms of drag, lift and axial forces with a CFD approach. The numerical simulation was performed with the software OpenFOAM ${ }^{\circledR} \mathrm{O}$ to be able to parallelize significantly the calculations reducing the computational time. The simulations shown that, at a reference speed of $60 \mathrm{~m} / \mathrm{s}$, the drag force can be significantly reduced by introducing a DSKW design. The wider lateral surface of the solid disk design produces, however, higher axial forces. These may reduce the drivability and manoeuvrability. At higher speed, the DSKW design seems to introduce significant lift effects.

The MGD6 design seems to be not competitive except for low speeds where the lift is reduced.

Further investigations are planned in order to complete this initial screening. 


\section{REFERENCES}

[1] Kyle, C.R., Selecting cycling equipment. In High Tech Cycling, ed. E.R. Burke, 2nd edn., Human Kinetics, pp. 1-48, 2003.

[2] Lukes, R.A., Chin, S.B. \& Haake, S.J., The understanding and development of cycling aerodynamics. Sports Engineering, 8(2), pp. 59-74, 2005. https://doi.org/10.1007/ bf02844004

[3] Greenwell, D.I., Wood, N.J., Bridge, E.K.L. \& Add, R.J., Aerodynamic characteristics of low-drag bicycle wheels. Journal of the Aeronautical Sciences, 99(983), pp. 109-120, 1995.

[4] Zdravkovich, M.M., Aerodynamics of bicycle wheel and frame. Journal of Wind Engineering and Industrial Aerodynamics, 40(1), pp. 55-70, 1992. https://doi. org/10.1016/0167-6105(92)90520-k

[5] Tew, G.S. \& Sayers, A.T., Aerodynamics of yawed racing cycle wheels. Journal of Wind Engineering and Industrial Aerodynamics, 82(1-3), pp. 209-222, 1999. https:// doi.org/10.1016/s0167-6105(99)00034-3

[6] Kyle, C.R. \& Burke, E., Improving the racing bicycle. Mechanical Engineering, 106(9), pp. 34-35, 1984.

[7] Kyle, C.R., Aerodynamic wheels. Bicycling, pp. 121-124, 1985.

[8] Kyle, C.R., New aero wheel tests. Cycling Science, 3(1), pp. 27-32, 1991.

[9] Fackrell, J.E. \& Harvey, J.K., The flowfield and pressure distribution of an isolated road wheel, advances on road vehicle aerodynamics. Fluid Engineering, ed. H.S. Stephens, BHRA, pp. 155-165, 1973.

[10] Fackrell, J.E., The Aerodynamics of an Isolated Wheel Rotating in Contact with the Ground, Ph.D. Thesis, University of London, London, U.K., 1974.

[11] Fackrell, J.E. \& Harvey, J.K., The aerodynamics of an isolated road wheel. In Proc. of 2nd AIAA Symposium of Aerodynamics of Sports and Competition Automobiles, ed. B. Pershing, LA, Calif., USA, pp. 119-125, 1975.

[12] Wray, J., A CFD Analysis into the Effect of Yaw Angle on the Flow Around an Isolated Rotating Wheel. Ph.D. Thesis, Cranfield University, U.K., 2003.

[13] McManus, J. \& Zhang, X., A computational study of the flow around an isolated wheel in contact with the ground. Journal of Fluids Engineering, 128(3), pp. 520-530, 2006. https://doi.org/10.1115/1.2175158

[14] Godo, M.N., Corson, D. \& Legensky, S.M., An aerodynamic study of bicycle wheel performance using CFD. 47th AIAA Aerospace Sciences Annual Meeting, Orlando, FL, USA, 5-8 January, 2009, AIAA Paper No. 2009-0322.

[15] Godo, M.N., Corson, D. \& Legensky, S.M., A comparative aerodynamic study of commercial bicycle wheels using CFD. 48th AIAA Aerospace Sciences Annual Meeting, Orlando, FL, USA, 4-7 January, 2010, AIAA Paper No. 2010-1431.

[16] Concli, F., Gobbi, M. \& Gorla, C., Comparative study of the aerodynamic performances of motorcycle racing wheels using numerical cfd simulations. WIT Transactions on Engineering Sciences, 120, pp. 185-191, 2018. https://doi.org/10.2495/afm180181

[17] OpenFOAM ${ }^{\circledR}$, www.openfoam.com

[18] Concli, F. \& Gorla, C., Windage, churning and pocketing power losses of gears: different modeling approaches for different goals. Forschung im Ingenieurwesen/Engineering Research, 80(3-4), pp. 85-99, 2016. https://doi.org/10.1007/s10010-016-0206-9

[19] Concli, F. \& Gorla, C., Numerical modeling of the churning power losses in planetary gearboxes: An innovative partitioning-based meshing methodology for the applica- 
tion of a computational effort reduction strategy to complex gearbox configurations. Lubrication Science, 29(7), pp. 455-474, 2017. https://doi.org/10.1002/1s.1380

[20] Concli, F., Gorla, C., Della Torre, A. \& Montenegro, G., Churning power losses of ordinary gears: A new approach based on the internal fluid dynamics simulations. Lubrication Science, 27(5), pp. 313-326, 2015. https://doi.org/10.1002/ls.1280

[21] Concli, F. \& Gorla, C., Analysis of the oil squeezing power losses of a spur gear pair by mean of CFD simulations. ASME 2012 11th Biennial Conference on Engineering Systems Design and Analysis, ESDA 2012(2), pp. 177-184, 2012.

[22] Concli, F., Gorla, C., Stahl, K., Höhn, B.R., Michaelis, K., Schultheiß, H. \& Stemplinger, J.P., Load independent power losses of ordinary gears: Numerical and experimental analysis. 5th World Tribology Congress, WTC 2013, pp. 1243-1246, 2013.

[23] Concli, F., Della Torre, A., Gorla, C. \& Montenegro, G., A new integrated approach for the prediction of the load independent power losses of gears: Development of a meshhandling algorithm to reduce the cfd simulation time. Advances in Tribology, 2016, art. no. 2957151.

[24] Concli, F. \& Gorla, C., Numerical modeling of the power losses in geared transmissions: Windage, churning and cavitation simulations with a new integrated approach that drastically reduces the computational effort. Tribology International, 103, pp. 58-68, 2016. https://doi.org/10.1016/j.triboint.2016.06.046

[25] Concli, F. \& Gorla, C., Influence of lubricant temperature, lubricant level and rotational speed on the churning power loss in an industrial planetary speed reducer: computational and experimental study. International Journal of Computational Methods and Experimental Measurements, 1(4), pp. 353-366, 2013. https://doi.org/10.2495/cmemv1-n4-353-366

[26] Concli, F., Thermal and efficiency characterization of a low-backlash planetary gearbox: An integrated numerical-analytical prediction model and its experimental validation. Proceedings of the Institution of Mechanical Engineers, Part J: Journal of Engineering Tribology, 230(8), pp. 996-1005, 2016. https://doi.org/10.1177/1350650115622363

[27] Rasedul Islam, Md., et al, Drag reduction of a car by using vortex generator. International Journal of Scientific \& Engineering Research, 4(7), pp. 1298-1302, 2013.

[28] Le niewicz, P., Kulak, M. \& Karczewski, M., Vehicle wheel drag coefficient in relation to travelling velocity-CFD analysis. Journal of Physics: Conference Series, 760(1), p. 012014, 2016. https://doi.org/10.1088/1742-6596/760/1/012014 